\title{
Structure of a soft-sphere fluid at a soft repulsive wall: A comparison of weighted density-functional theories
}

\author{
Rachel Sibug-Aga and Brian B. Laird* \\ Department of Chemistry, University of Kansas, Lawrence, Kansas 66045, USA
}

(Received 16 June 2003; published 26 May 2004)

\begin{abstract}
Density-functional theory is used to investigate the structure of a soft-sphere fluid at a soft wall. The fluid is modeled by an inverse sixth-power repulsive pair potential and the fluid particles interact with a flat stationary wall defined by an inverse-twelth power repulsive external potential. For comparison we examine results using three weighted density approximations (WDA), namely those due to Curtin and Ashcroft [Phys. Rev. A 32, 2909 (1985)], Denton and Ashcroft [Phys. Rev. A 39, 426 (1989)], and the partitioned WDA of Kol and Laird [Mol. Phys. 90, 951 (1997)]. The degree to which each of these approximations can accurately predict the structure of the fluid at the wall is evaluated for several densities through comparison with Monte Carlo simulation data.
\end{abstract}

DOI: 10.1103/PhysRevE.69.051502

PACS number(s): 61.20.Gy, 61.20.Ne, 61.20.Ja, 68.08.De

\section{INTRODUCTION}

In recent years, there has been considerable interest in the formulation of density-functional theories (DFT's) to predict the structure and thermodynamics of inhomogeneous fluids, with applications in colloid and interface science, electrochemistry, and a variety of other fields. Fluids that have a special geometrical symmetry or those fluids in confined geometries have been the commonly used models, the simplest of which is that of a fluid at a structureless wall. DFT studies involving such geometry have been performed on one- [1-3] and two-component [4,5] hard spheres, as well as on Lennard-Jones fluids [6]. Other similar configurations of interest are a fluid near a hard cylinder [1], a fluid confined in a spherical cavity [7], and a fluid around a large hard sphere [7]. The vast majority of DFT studies of confined fluids have focused on systems with short ranged repulsions such as the hard sphere and Lennard-Jones systems.

The fluid/wall geometry is significant in solid-liquid interface studies since a fluid at a structureless wall can provide a first approximation to a heterogeneous solid-liquid interface, where the structure of the solid does not contribute significantly to the fluid structure developed within the interfacial region. The advantage is that the problem is considerably simplified, yet, the generic features of the structure of the fluid within the interfacial region are obtained. Since this type of approximation does not consider the actual solid, it is then possible to isolate the effects of particle interactions between the real solid and the fluid. A drawback of the model is that there is no information about the solid that can be obtained.

Most classical DFT's fall into three classes: (a) perturbative methods based on functional Taylor-series expansions about some reference state [8,9]—usually the homogeneous phase, (b) nonperturbative methods based on weighted density-functional theories (WDFT) [11-14], or (c) methods based on the fundamental measure theory (FMT) of Rosen-

\footnotetext{
*Electronic address: blaird@ku.edu
}

feld [15]. For predicting the structure and thermodynamics of hard-sphere inhomogeous fluids, the highest accuracy theories are those based on fundamental measure theory. Recently, soft-sphere versions of that FMT formalism have been developed $[16,17]$. While these methods are important first steps in the development of a general FMT for soft spheres, they are not applicable to the systems of interest in this work - namely those with long range repulsive cores. The method outlined by Schmidt [16] diverges above a density of $0.26 \sigma^{-3}$ for the inverse-sixth power potential studied here, whereas the " $\sigma$-cavity" FMT of Sweatman [17] is designed for systems with a finite range $\sigma$, but no procedure is given for the determination (or meaning) of this range for purely repulsive inverse power systems. In the absence of applicable FMT theories, weighted DFT's are the current best candidates for longer ranged repulsive potentials.

The development of weighted density approximations (WDA's) of the free energy functional for classical inhomogeneous fluids was initiated by Nordholm [10] and Tarazona [11]. A number of formulations have since been developed and tested for hard spheres, and most have demonstrated good agreement with Monte Carlo simulations - see, for example, the comparison of different WDA methods for a hard sphere fluid at a wall reported by Kroll and Laird [2]. In addition, systems with long range attractive interactions and short range repulsive cores, such as Lennard-Jones, have been successfully treated by combining a perturbative treatment of the attraction with a hard-sphere WDA approximation for the repulsion [18]. However, the application of WDA methods to inhomogeneous fluids with longer range repulsive cores, on the other hand, have not received much attention. In this study, we examine the structure of a simple model soft-sphere fluid at a soft wall as calculated by three different weighted density approximation schemes, namely, that of Curtin and Ashcroft (CA WDA) [12], a simplified WDA of Denton and Ashcroft (DA WDA) [14], and the partitioned WDA of Kol and Laird (KL WDA) [13]. The specific model system we study is an inverse-sixth power repulsive fluid against a stationary, structureless wall defined by a repulsive inverse twelth-power external potential. Results for 
these three DFT's at a variety of bulk densities are compared with Monte Carlo simulations.

\section{WEIGHTED DENSITY-FUNCTIONAL THEORIES OF INHOMOGENEOUS FLUIDS}

Density-functional theories have their origin in a theorem due to Hohenberg and Kohn [19], which was extended to the finite temperature case by Mermin [20]. For a system at fixed temperature, $T$, chemical potential, $\mu$, and external singleparticle potential, $\phi(\mathbf{r})$, Mermin proved the existence of a functional $\mathcal{F}[\rho(\mathbf{r})]$, independent of $u(\mathbf{r})$ and $\mu$, such that the functional

$$
\Omega[\rho(\mathbf{r})]=\mathcal{F}[\rho(\mathbf{r})]+\int d \mathbf{r} \rho(\mathbf{r})[u(\mathbf{r})-\mu]
$$

is a minimum for the correct equilibrium single-particle density $\rho(\mathbf{r})$ subject to the external potential. The value of $\Omega$ at this minimum is the value of the grand potential. The density that minimizes Eq. (1) is determined by either numerically solving the Euler-Lagrange equation

$$
\frac{\delta \Omega}{\delta \rho(\mathbf{r})}=0
$$

or by parameterizing $\rho(\mathbf{r})$ and minimizing Eq. (1) with respect to the parameters.

For a classical system [21], the functional $\mathcal{F}[\rho]$ can be written as the sum of an ideal part, $\mathcal{F}_{i d}[\rho]$, and an excess part, $\mathcal{F}_{e x}[\rho]$, due to the interparticle interactions:

$$
\mathcal{F}[\rho]=\mathcal{F}_{i d}[\rho]+\mathcal{F}_{e x}[\rho] .
$$

For a classical monatomic system, the ideal part is known exactly and is given by

$$
\beta \mathcal{F}_{i d}[\rho]=\int d \mathbf{r} \rho(\mathbf{r})\left\{\ln \left[\Lambda^{3} \rho(\mathbf{r})\right]-1\right\},
$$

where $\Lambda$ is the thermal wavelength, and $\beta=(k T)^{-1}$. In general, the excess part, $\mathcal{F}_{e x}$ is unknown; therefore, the central task of a DFT method is to provide a suitable approximation for this quantity. The quantity $\mathcal{F}_{e x}$ is related to the structure of an inhomogeneous fluid through the hierarchy of $n$-particle direct correlation functions, $c^{(n)}\left(\mathbf{r}_{1}, \ldots, \mathbf{r}_{n} ;[\rho(\mathbf{r})]\right)$ :

$$
\frac{\delta^{n} \beta \mathcal{F}_{e x}}{\delta \rho\left(\mathbf{r}_{1}\right) \ldots \delta\left(\mathbf{r}_{n}\right)}=c^{(n)}\left(\mathbf{r}_{1}, \ldots, \mathbf{r}_{n} ;[\rho(\mathbf{r})]\right) ;
$$

this hierarchy is often useful in the development of DFT approximation schemes.

Classical density-functional theories using a weighted density approach were first introduced by Nordholm, Johnson, and Freasier [10], and Tarazona [11]. Such theories are modifications of the usual local density approximation (LDA) for inhomogeneous systems. In the LDA, the free energy density at a point $\mathbf{r}$ in a system with inhomogeneous single-particle density $\rho(\mathbf{r})$ is given by the free energy per particle of a homogeneous system, evaluated at the value of the single-particle density at point $\mathbf{r}$

$$
\mathcal{F}_{e x}=\int_{V} d \mathbf{r} \rho(\mathbf{r}) f_{0}\left(\hat{\rho}_{2}\right) .
$$

However, for very strongly inhomogeneous systems the LDA breaks down. To remedy this, the local density is averaged over a small region using a weighting function $w\left(\mid \mathbf{r}_{1}\right.$ $\left.-\mathbf{r}_{2} \mid ; \hat{\rho}\right)$ to create a coarse-grained or "weighted" density $\hat{\rho}(\mathbf{r})$ :

$$
\hat{\rho}\left(\mathbf{r}_{1}\right)=\int d \mathbf{r}_{2} \rho\left(\mathbf{r}_{2}\right) w\left(\left|\mathbf{r}_{1}-\mathbf{r}_{2}\right| ; \hat{\rho}\left(r_{1}\right)\right) .
$$

A specific weighted DFT is defined by specifying a procedure by which $w(r, \rho)$ is determined given input as to the properties of the system. In this work we compare three different weighted density-functional theories: the weighted density approximation of Curtin and Ashcroft (CA WDA) [12], a simplified version of the CA WDA by Denton and Ashcroft (DA WDA) [4,14], and the partitioned weighted density approximation of Kol and Laird (KL WDA) [13]. All of these methods require as input the excess Helmholtz free energy per particle $f(\rho)$ and the two-body direct correlation function of the homogeneous fluid $c(r ; \rho)$, both of which can be obtained from experiment, simulation, or integral equation theory [22].

\section{A. Weighted density approximation of Curtin and Ashcroft (CA WDA)}

In this approach, the excess free energy functional of the inhomogeneous system is approximated by that of a homogeneous system evaluated at a weighted density, $\hat{\rho}(\mathbf{r})$,

$$
F_{e x}[\rho]=\int d \mathbf{r} f(\hat{\rho}(\mathbf{r})) \rho(\mathbf{r}),
$$

where $f(\rho)$ is the excess free energy per particle of the homogeneous fluid as a function of the bulk density $\rho$ and $\hat{\rho}$ is as defined in Eq. (7) above.

Within the CA WDA [12], the weight function is determined by requiring that the second functional derivative of $\mathcal{F}_{\text {ex }}$ reproduce the correct value of the two-particle direct correlation function, $c^{(2)}$, for the liquid in the homogeneous limit,

$$
c^{(2)}\left(\left|\mathbf{r}_{2}-\mathbf{r}_{1}\right| ; \rho_{0}\right)=-\lim _{\rho\left(\mathbf{r}_{2}\right) \rightarrow \rho_{0}} \frac{\delta^{(2)} \beta F_{e x}[\rho]}{\delta \rho\left(\mathbf{r}_{1}\right) \delta \rho\left(\mathbf{r}_{2}\right)} .
$$

Using (8) and the normalization requirement that $\int d \mathbf{r}_{2} w\left(\mid \mathbf{r}_{2}\right.$ $\left.-\mathbf{r}_{1} \mid ; \hat{\rho}\left(\mathbf{r}_{1}\right)\right)=1$, the following differential equation for $w(r)$, in terms of $c(r ; \rho)$ and $f(\rho)$, is obtained [12]:

$$
\begin{aligned}
c^{(2)}(k ; \rho)= & -2 \beta f^{\prime}(\rho) w(k ; \rho)-\beta \rho f^{\prime \prime}(\rho) w^{2}(k ; \rho) \\
& -2 \beta \rho f^{\prime}(\rho) w(k ; \rho) \partial w(k ; \rho) / \partial \rho .
\end{aligned}
$$

(For a detailed derivation of the above expression for $w$, the reader should consult Ref. [12].) This differential equation can be solved either by iteration [12] or by using a standard numerical differential equation solver [23].

The CA WDA has been used to successfully predict the freezing transition of hard spheres [18] and Lennard-Jones 
fluids [18], the structure and interfacial free energy of the hard-sphere crystal-melt interface [24,25], as well as the structure and thermodynamics of hard spheres at a hard wall [2]. A simplification of the CA WDA, the modified weighteddensity approximation (MWDA) of Denton and Ashcroft [26] has also been successful in predicting the freezing of hard spheres. However, when the MWDA is applied to the longer ranged inverse-sixth repulsive system, the method fails to predict a stable bcc structure [27].

\section{B. Weighted density approximation of Denton and Ashcroft (DA WDA)}

Due to its complexity, calculations using the CA WDA functional can be difficult to implement successfully. A simpler alternative is the DA WDA [4,14]. In this approximation a weighted density approximation is applied not to the excess free energy functional but to its functional derivative with respect to $\rho(\mathbf{r})$, namely the single particle direct correlation function as defined in Eq. (5) (with $n=1$ ).

Using Eqs. (3)-(5), functional minimization of Eq. (1) leads to a formally exact expression for the inhomogeneous single-particle density

$$
\rho(\mathbf{r})=\rho_{0} \exp \left\{-\beta u(\mathbf{r})+c^{(1)}(\mathbf{r} ;[\rho])-c_{0}^{(1)}\left(\rho_{0}\right)\right\},
$$

where $c_{0}^{(1)}\left(\rho_{0}\right)$ is the single-particle direct correlation function of a homogeneous fluid, evaluated at the bulk density $\rho_{0}$. In the DA WDA, the approximation is made such that $c^{(1)}(\mathbf{r} ;[\rho])$ in the equation above is replaced by its homogeneous counterpart evaluated at a weighted density as defined in Eq. (7):

$$
c^{(1)}(\mathbf{r} ;[\rho])=c_{0}^{(1)}(\hat{\rho}(\mathbf{r})) .
$$

The procedure for obtaining the weight function used for the calculation of $\hat{\rho}(\mathbf{r})$ makes use of the expressions for the one- and two- particle direct correlation functions in terms of the derivatives of the excess free energy. With (5) and (12), the two-particle direct correlation function in the homogeneous limit can be expressed in the form

$$
c_{0}^{(2)}\left(\left|\mathbf{r}_{2}-\mathbf{r}_{1}\right| ; \rho_{0}\right)=\lim _{\rho\left(\mathbf{r}_{2}\right) \rightarrow \rho_{0}} \frac{\delta c^{(1)}(\mathbf{r} ;[\rho])}{\delta \rho\left(\mathbf{r}_{2}\right)} .
$$

From this relation, an equation for the weight function is derived,

$$
w\left(r ; \rho_{0}\right)=\frac{c_{0}^{(2)}\left(r ; \rho_{0}\right)}{\partial c_{0}^{(1)}\left(\rho_{0}\right) / \partial \rho_{0}} .
$$

With this approximation for $c^{(1)}(\mathbf{r} ;[\rho])$, Eq. (11) can be solved self-consistently for a specific system until convergence is obtained. Note that, since the DA WDA does not have a unique associated free energy, it is limited to structural studies (such as the present application) and would not be useful, for example, in the identification of phase transitions.

\section{Partitioned weighted density approximation of Kol and Laird (KL WDA)}

Although the CA WDA (and the related MWDA of Denton and Ashcroft) have been shown to adequately predict the fluid/fcc crystal freezing transition for hard spheres [12,26] and Lennard-Jones particles [18], it fails completely to predict freezing into nonclose-packed structures of longer ranged potentials, for example the fluid to bcc transition for particles interacting with an inverse-sixth-power repulsive pair potential [27]. To remedy this, Kol and Laird [13] have demonstrated that if the excess free energy functional is partitioned into long and short range parts and only the short range part of the free energy is approximated by a MWDA scheme, the prediction of a stable bcc structure for the inverse sixth-power potential is realized. Motivated by these results, we have reformulated this partitioned WDA of Kol and Laird (KL WDA) within the context of CA WDA in order to apply it to current inhomogeneous fluid problem. The difficulty of implementation of this method is roughly comparable to that for the CA WDA.

In principle, the free energy functional can be partitioned into entropic and energetic contributions as given below,

$$
\begin{aligned}
\mathcal{F}_{e x}[\rho]= & -T S_{e x}[\rho]+\epsilon_{e x}[\rho] \\
= & -T S_{e x}[\rho]+\frac{1}{2} \iint d \mathbf{r}_{1} d \mathbf{r}_{2} \phi\left(r_{12}\right) \rho\left(\mathbf{r}_{1}\right) \rho\left(\mathbf{r}_{2}\right) \\
& \times g^{(2)}\left(\mathbf{r}_{1}, \mathbf{r}_{2} ;[\rho]\right),
\end{aligned}
$$

where $g^{(2)}\left(\mathbf{r}_{1}, \mathbf{r}_{2} ;[\rho]\right)$ is the pair correlation function for an inhomogeneous system. In partitioned WDA [13], the partitioning given in Eq. (15) is not exactly followed because the function, $g^{(2)}\left(\mathbf{r}_{1}, \mathbf{r}_{2} ;[\rho]\right)$, is not known, thus making the construction of an explicit expression for the free energy functional difficult. Instead, the excess free energy functional is divided into short and long range parts, which are denoted as $\mathcal{F}_{1}[\rho]$ and $\mathcal{F}_{2}[\rho]$, respectively, in the equation below.

$$
\mathcal{F}_{\text {ex }}[\rho]=\mathcal{F}_{1}[\rho]+\mathcal{F}_{2}[\rho],
$$

where the long range functional $\mathcal{F}_{2}$ is defined as follows

$$
\mathcal{F}_{2}[\rho]=\frac{1}{2} \iint d \mathbf{r}_{1} d \mathbf{r}_{2} \phi\left(r_{12}\right) \rho\left(\mathbf{r}_{1}\right) \rho\left(\mathbf{r}_{2}\right) \theta\left(r_{12} ; l\right) .
$$

The switching function $\theta\left(r_{12} ; l\right)$ is a crude approximation to the actual $g^{(2)}\left(\mathbf{r}_{1}, \mathbf{r}_{2} ;[\rho]\right)$ and is chosen here to be

$$
\theta\left(r_{12} ; l\right)=\exp \left[-(l / r)^{12}\right]
$$

where $l$ can be roughly interpreted physically as a value of $r$ beyond which $c(r) \approx-\beta \phi(r)$ is a reasonable approximation. (The form of the switching function was chosen to give a smoothed step function - the use of a discontinuous step function was found in the earlier work [13] to lead to unacceptable discontinuities in the functional.) The short ranged part, $\mathcal{F}_{1}$ is primarily entropic in nature and is approximated using a weighted-density functional scheme 


$$
\mathcal{F}_{1}=\int_{V} d \mathbf{r} \rho(\mathbf{r}) f_{1}(\hat{\rho}(\mathbf{r}))
$$

where $f_{1}(\rho)$ is obtained from the full excess free energy for the homogeneous fluid by subtracting off the contribution from $\mathcal{F}_{2}$ (evaluated in the homogeneous limit). In contrast, the long ranged part, $\mathcal{F}_{2}[\rho]$ can be calculated exactly, and is primarily energetic. The results for this method do depend on the choice of the value of the switching length, $l$, for a given density (obviously, as $l \rightarrow \infty$ the CA WDA limit obtains), so it is necessary to have a criterion with which to choose the switching length. In the earlier work of of Kol and Laird the value of $l$ was chosen so that the free energy was minimum; however, in the present application, no minimum was detected and it was found that an operative criterion was to choose the minimum value of $l$ for which solutions for the weight functions could be obtained. (Since there is no variational theorem for the dependence of the free energy on $l$, there is no physical reason to choose one criterion over the other - that choice has to be made on a case by case basis and is admittedly ad hoc). Both of these criteria lead to values of $l$ that are density dependent, which has the drawback that the Gibbs adsorption equation is not satisfied for such methods $[28,29]$; however, since we are interested only in the prediction of structure in the current work, this is not an issue. In situations where the validity of the Gibbs adsorption equation is important, one can still use the KL WDA by fixing $l$ at some intermediate value appropriate for the density range studied. (Of course, this assumes that it is possible to choose a reasonable value of $l$ that gives solutions over density range of interest for the given system of study, a fact that cannot be guaranteed a priori.)

With the partitioning of the free energy and with the expression given in Eq. (17), an expression for the two-particle direct correlation function in the homogeneous limit is

$$
\begin{aligned}
c^{(2)}\left(\left|\mathbf{r}_{2}-\mathbf{r}_{1}\right| ; \rho_{0}\right)= & \Delta c^{(2)}\left(\left|\mathbf{r}_{2}-\mathbf{r}_{1}\right| ; \rho_{0}\right) \\
& -\beta \phi\left(\left|\mathbf{r}_{2}-\mathbf{r}_{1}\right|\right) \theta\left(\left|\mathbf{r}_{2}-\mathbf{r}_{1}\right| ; l\right) .
\end{aligned}
$$

The function $\Delta c^{(2)}\left(\left|\mathbf{r}_{2}-\mathbf{r}_{1}\right| ; \rho_{0}\right)$ is shorter ranged than the two-particle direct correlation function. We then use this quantity and the short ranged free energy to find the weight function from the condition that

$$
\Delta c^{(2)}\left(\left|\mathbf{r}_{2}-\mathbf{r}_{1}\right| ; \rho_{0}\right)=-\lim _{\rho\left(\mathbf{r}_{2}\right) \rightarrow \rho_{0}} \frac{\delta^{(2)} \beta F_{1}[\rho]}{\delta \rho\left(\mathbf{r}_{1}\right) \delta \rho\left(\mathbf{r}_{2}\right)} .
$$

The weight function is found by using an equation identical to that for the CA WDA [Eq. (10)] with $\Delta c^{(2)}$ replacing $c^{(2)}$. As mentioned above, the value of the parameter $l$ was chosen as the minimum value for which a solution for $w\left(k ; \rho_{0}\right)$ can be obtained. The weight functions are similar in form and range to the weight functions from the CA WDA method applied to the hard-sphere system [13]. The weight functions for hard spheres exhibit a discontinuity at the sphere diameter, which is also the range of the hard-sphere interaction.

\section{RESULTS FOR A SOFT-SPHERE FLUID AT A SOFT REPULSIVE WALL}

To compare the three weighted density approximations, we examine the structure of a simple model soft-sphere fluid at a soft repulsive wall. The fluid consists of particles interacting via an inverse sixth-power repulsive pair potential:

$$
\phi(r)=\epsilon(\sigma / r)^{6},
$$

where $r$ is the interparticle separation. (Note that, for inverse $n$-power potentials excess thermodynamic quantities are only a function of $\left.\gamma_{n}=\rho \sigma^{3}(k T / \epsilon)^{-3 / n}=\rho^{*} T^{*-3 / n}\right)$. This potential is a good candidate for the study of the structure of longer-ranged repulsive potentials in that it is (a) simple and (b) not well approximated by a hard-sphere model - for example, this system freezes into a bcc crystal structure $[30,31]$ - such structures are mechanically unstable for shorter ranged potentials such as hard spheres or LennardJones spheres. The system also exhibits a bcc to fcc solidsolid phase transition as the density is raised (or the temperature is lowered) beyond the freezing point. This phase diagram is qualitatively similar to bcc forming metals, such as iron, and the inverse-power system is a useful simple model for the study of generic phase behavior of such metals.

The soft-sphere fluid interacts with a stationary, structureless soft wall defined by an external field given by

$$
u(z)=\epsilon(\sigma / z)^{12},
$$

where $z$ is the distance between the wall and a fluid particle along the direction normal to the interfacial plane.

In this study the DFT calculations are performed at a reduced temperature of $T^{*}=k T / \epsilon=0.1$ and for three different reduced bulk densities $\rho_{0}^{*}=\rho_{0} \sigma^{3}=0.15,0.30$, and 0.50 , respectively. At this temperature, the reduced freezing density is 0.69 [31]. For all DFT's studied, the excess free energy $f(\rho)$ and pair direct correlation function $c^{(2)}(r)$ for the homogeneous inverse sixth-power fluid are needed as input. This input liquid structure and thermodynamic data was calculated using the modified hypernetted-chain integral equation method [32-34]. For details of the specific version used, see the article by Rosenfeld [35]. The integral equation was solved numerically using the method of Lábik, Malijevský, and Vońka [36]. This integral equation gives results for this system that are in excellent agreement with computer simulation [27].

The procedure used to determine the density profiles for this wall-fluid problem using the CA WDA and KL WDA is as follows.

(1) Equation (10) (or its analog for KL WDA) is solved iteratively to give $w(k ; \rho)$ (using as initial value the solution to the quadratic equation obtained by setting the last term on the right-hand side of Eq. (10) to zero).

(2) For the given bulk density $\rho$, the corresponding chemical potential is determined from the homogeneous excess free energy by

$$
\mu=\ln \rho+\rho f^{\prime}(\rho)+f(\rho) .
$$




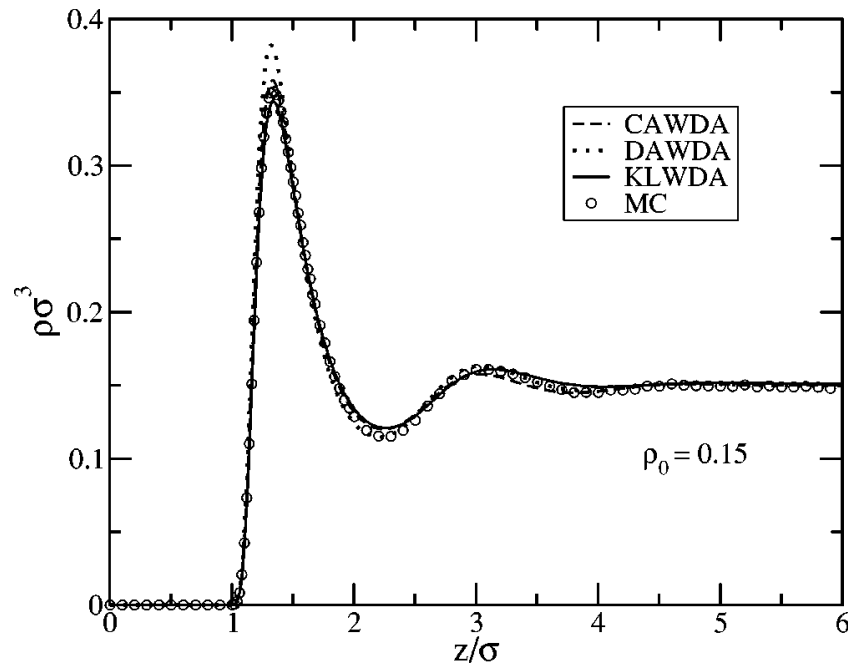

FIG. 1. Density profiles $\rho(z)$ for $\rho_{0}=0.15 / \sigma^{3}$ calculated using the three WDA methods in comparison with $\mathrm{MC}$ simulation (circles).

(3) Minimize Eq. (1) with respect to $\alpha(z)$ defined by

$$
\rho(z)=\rho_{0} e^{-\beta u(z)} e^{\alpha(z)} .
$$

(4) The "ideal" (noninteracting or low-density) solution $\rho \exp [-\beta u(z)]$ has been factored out here to simplify the parameterization. The minimization for both the CA WDA and KL WDA is performed using the Fletcher-Reeves-PolakRibiere conjugate gradient algorithm [23] with a uniform grid of $2048 z$ points with spacing $d z=0.005 \sigma$ and an initial guess of $\alpha=0$ (as a test minimization was also performed using results from the DA WDA or MC simulations as initial guesses and no change in the final solution was seen).

For the DA WDA, Eq. (11) is solved iteratively using an initial guess of $\alpha(z)=0$ and the same $z$ grid as for the other two methods. The values of $l$ used to construct $\Delta c^{(2)}(r)$ for the KL WDA are $1.15 \sigma, 1.05 \sigma$, and $0.95 \sigma$ for $\rho^{*}=0.15,0.30$, and 0.50 , respectively

For comparison we have also performed standard Metropolis Monte Carlo simulations on each of these systems, using 500, 700, and 700 particles, respectively, for $\rho_{0}^{*}=0.15$, 0.30 , and 0.50 . Each simulation was equilibrated from a random configuration for $8 \times 10^{6}$ cycles followed by $2 \times 10^{6}$ cycles to collect averages. The box sizes $\left(L_{x} / \sigma, L_{y} / \sigma, L_{z} / \sigma\right)$ used for these three runs, respectively, are $(14.8,14.8,16.8)$, $(11.7,11.7,18.3),(7.9,7.9,23.7)$, for $\rho^{*}=0.15,0.30$, and 0.50 - the box sizes were chosen so as to give the correct bulk density away from the wall. Two additional MC runs for the system at $\rho_{0}^{*}=0.50$ with 1400 particles were run - one in which the number of particles was doubled by doubling $L_{z}$ and keeping the $x-y$ cross-sectional area fixed, and one in which the cross-sectional area was doubled, keeping $L_{z}$ fixed. The results for the larger systems showed no detectable size dependence in the density profiles.

Figures 1-3 show density profiles for $\rho_{0}=0.15 / \sigma^{3}, \rho_{0}$ $=0.30 / \sigma^{3}$, and $\rho_{0}=0.50 / \sigma^{3}$, respectively. All of the methods studied predict the position of the first peak at each density with reasonable accuracy at the lower densities $(0.30$ and

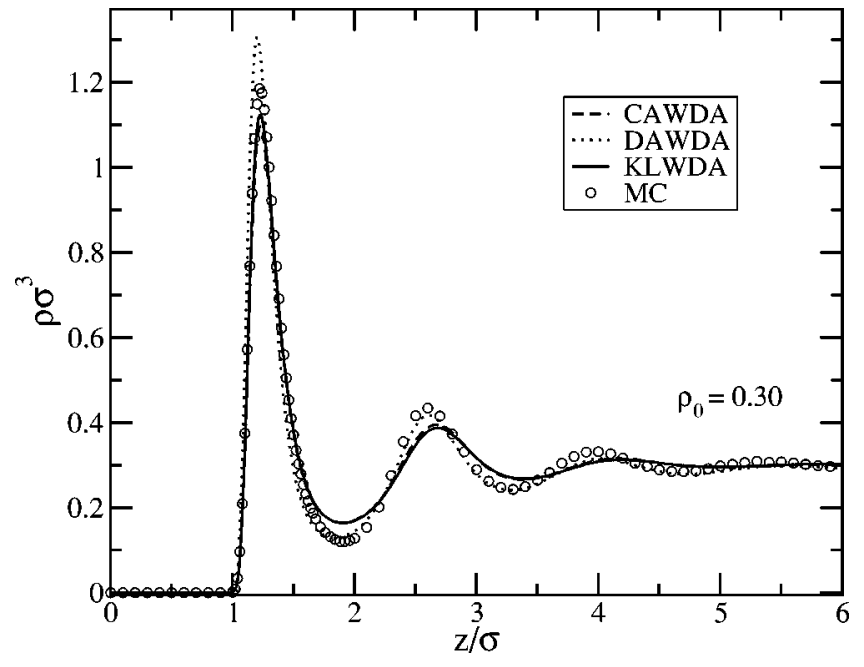

FIG. 2. Same as Fig. 1 except with $\rho_{0}=0.30 / \sigma^{3}$.

0.50), although, the KL WDA and CA WDA show better agreement with Monte Carlo results for the height of the first density peak as compared to the DA WDA method, which significantly overestimates the height of the first density peak. This limitation of the DA WDA method has also been observed when applied to hard spheres against a hard wall and is a consequence of a failure to satisfy the pressure sum rule [4]. However, at the lowest densities the DA WDA is seen to give surprisingly good agreement with simulation beyond the first peak - even better than the other two, much more involved methods. At the highest density (0.50), the best method is the KL WDA, which accurately predicts the position and height of all density peaks. It should be noted, that despite the differences, the agreement with simulation of all three methods is good.

For the KL WDA, it is useful to examine the sensitivity of the method to the choice of the switching parameter $l$. Figure 4 shows the dependence of the predicted density profiiles

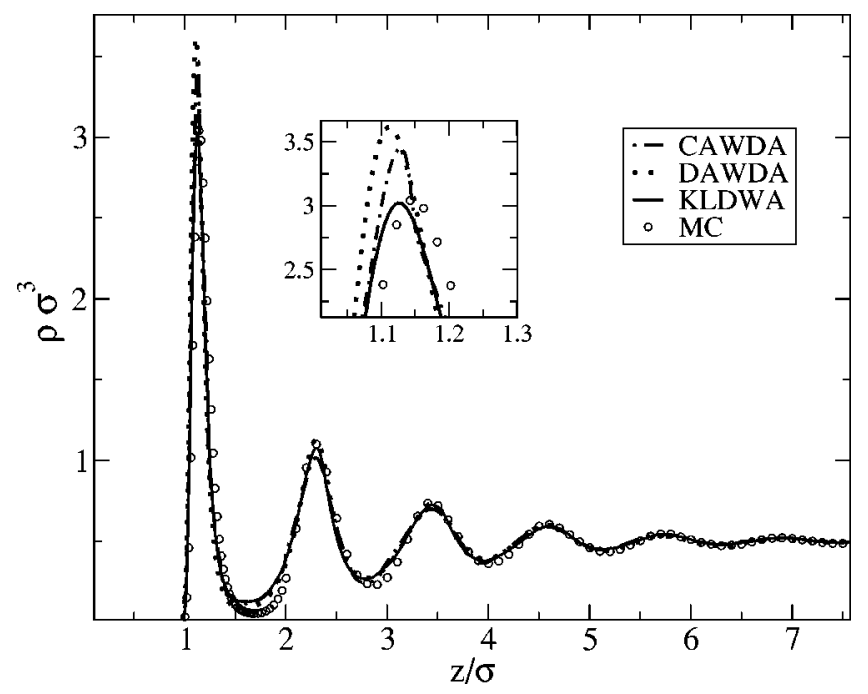

FIG. 3. Same as Fig. 1 except with $\rho_{0}=0.50 / \sigma^{3}$. For clarity, a magnification of the region around the first peak is shown in the inset. 


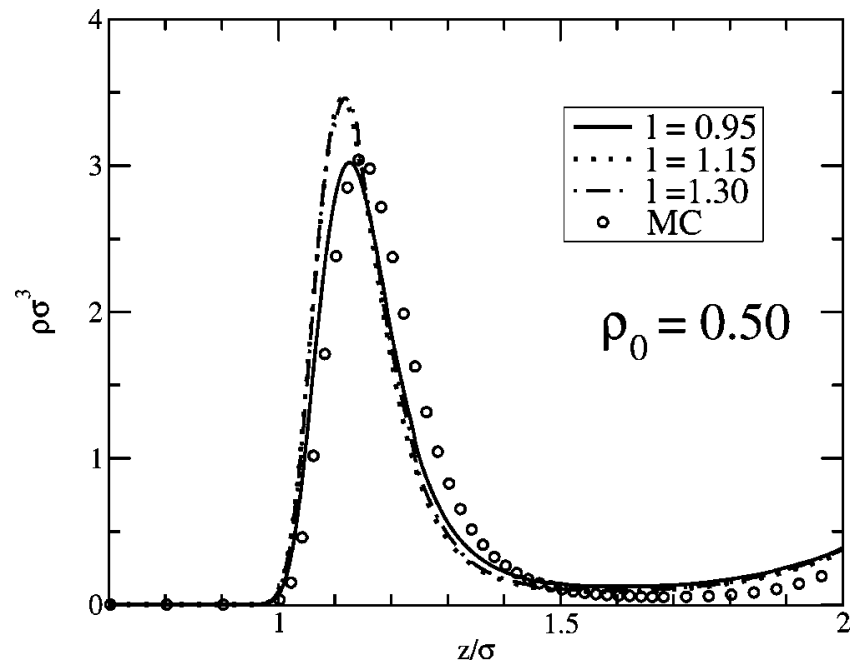

FIG. 4. Density profiles for $\rho_{0}=0.50 / \sigma^{3}$ as predicted by the KL WDA for different values of the switching parameter $l$. For comparison the MC simulation values are shown as open circles.

$\rho(z)$ for bulk density $\rho_{0}=0.50 / \sigma^{3}$ for three different values of $l / \sigma: 0.95,1.15$, and 1.30 . The solutions for the large values of $l$ are roughly identical to each other and to the CA WDA solution (which is the $l \rightarrow \infty$ limit of the KL WDA). It is only at $l=0.95 \sigma$ (the value that was used in the calculation) that differences are seen. At this value of $l$ there is significant improvement (relative to the higher values of $l$ ) of the first density peak in comparison to the MC simulation.

\section{SUMMARY}

To determine the accuracy to which classical densityfunctional techniques can predict the structure of inhomge- neous fluids for systems with long ranged repulsive interactions (i.e., with longer ranged repulsive cores than hardsphere or Lennard-Jones interactions), we have compared the results of three different WDA methods with Monte Carlo simulation for a system consisting of a model fluid of particles interacting through an inverse sixth-power repulsive pair potential at a soft wall. The three methods studied are the the WDA of Curtin and Ashcroft (CA WDA) [12], a simplified WDA of Denton and Ashcroft (DA WDA) [14], and the partitioned WDA of Kol and Laird (KL WDA) [13]. The predictions of the three methods are seen to be quite accurate when compared to Monte Carlo simulation. The DA WDA overestimates the height of the first peak, but for larger distances is more accurate than the other methods at low density. At high density, the KL WDA is seen to be the most accurate method for the first peak. That the KL WDA is better at predicting the structure of inhomogeneous fluids for longer range potentials than the CA WDA is not too surprising given earlier results showing that KL WDA [13] is more successful than the CA WDA at predicting the freezing transtion for inverse-power potentials. Finally, when considering these methods for the purposes of a specific application one should take into account the fact that the DA WDA is, by far, the easiest and least computationally intensive method to implement and run for these systems.

\section{ACKNOWLEDGMENT}

The authors gratefully acknowledge support from the National Science Foundation under Grant No. CHE9970903.
[1] R. D. Groot, N. M. Faber, and J. P. van Eerden, Mol. Phys. 62, 861 (1987).

[2] D. M. Kroll and B. B. Laird, Phys. Rev. A 42, 4806 (1990).

[3] E. Kierlik and M. L. Rosinberg, Phys. Rev. A 42, 3382 (1990).

[4] A. R. Denton and N. W. Ashcroft, Phys. Rev. A 44, 8242 (1991).

[5] R. Roth and S. Dietrich, Phys. Rev. E 62, 6926 (2000).

[6] M. B. Sweatman, Phys. Rev. E 65, 011102 (2002).

[7] M. Moradi et al., Phys. Rev. E 63, 021202 (2001).

[8] T. V. Ramakrishnan and M. Yussouff, Phys. Rev. B 19, 2775 (1979).

[9] A. D. J. Haymet and D. W. Oxtoby, J. Chem. Phys. 74, 2559 (1981).

[10] S. Nordholm, M. Johnson, and B. C. Freasier, Aust. J. Chem. 33, 2139 (1980).

[11] P. Tarazona, Mol. Phys. 52, 81 (1984).

[12] W. A. Curtin and N. W. Ashcroft, Phys. Rev. A 32, 2909 (1985).

[13] A. Kol and B. B. Laird, Mol. Phys. 90, 951 (1997).

[14] A. R. Denton and N. W. Ashcroft, Phys. Rev. A 39, 426 (1989).

[15] Y. Rosenfeld, Phys. Rev. Lett. 63, 980 (1989).
[16] M. Schmidt, Phys. Rev. E 62, 4976 (2000).

[17] M. B. Sweatman, J. Phys.: Condens. Matter 14, 11921 (2002).

[18] W. A. Curtin and N. W.Ashcroft, Phys. Rev. Lett. 56, 2775 (1986).

[19] P. Hohenberg and W. Kohn, Phys. Rev. 136, B864 (1964).

[20] N. D. Mermin, Phys. Rev. 137, A1441 (1965).

[21] W. F. Saam and C. Ebner, Phys. Rev. A 15, 2566 (1977).

[22] J. P. Hansen and I. R. McDonald, Theory of Simple Liquids, 2nd ed. (Academic, New York, 1986).

[23] W. H. Press, S. A. Teukolsky, W. T. Vetterling, and B. P. Flannery, Numerical Recipies in Fortran (Cambridge University Press, New York, 1992).

[24] W. A. Curtin, Phys. Rev. Lett. 59, 1228 (1987).

[25] W. A. Curtin, Phys. Rev. B 39, 6775 (1989).

[26] A. R. Denton and N. W. Ashcroft, Phys. Rev. A 39, 4701 (1989).

[27] B. B. Laird and D. M. Kroll, Phys. Rev. A 42, 4810 (1990).

[28] M. B. Sweatman, Mol. Phys. 98, 573 (2000).

[29] M. B. Sweatman, Phys. Rev. E 63, 031102 (2001).

[30] W. G. Hoover, M. Ross, K. W. Johnson, D. Henderson, J. A. Barker, and B. C. Brown, J. Chem. Phys. 52, 4931 (1970). 
[31] B. B. Laird and A. D. J. Haymet, Mol. Phys. 75, 71 (1992).

[32] Y. Rosenfeld and N. W. Ashcroft, Phys. Rev. A 20, 1208 (1979).

[33] F. Lado, Phys. Lett. 89, 196 (1982).

[34] F. Lado, S. M. Foiles, and N. W. Ashcroft, Phys. Rev. A 28 ,
2374 (1983).

[35] Y. Rosenfeld, J. Stat. Phys. 42, 437 (1986).

[36] S. Labik, A. Malijevsky, and P. Vonka, Mol. Phys. 56, 709 (1985). 